Vorwort zur vierten Auflage.

In der vorliegenden neuen Auflage wurden die Präparate aus der aliphatischen Reihe um die Darstellung von Glykol aus Äthylenbromid vermehrt. Herrn Prof. Dr. L. Henky in Löwen bin ich für wertvolle Ratschläge bezgl. dieses Präparates zu großem Danke verpflichtet. Unter die aromatischen Präparate wurde ein Beispiel für die Reimer-Tiemannsche Synthese (Salicylaldehyd), sowie die Zerlegung der inaktiven Mandelsäure in ihre altiven Komponenten neu aufgenommen. - Die alten Vorschriften konnten beibehalten werden, wurden jedoch in manchen Punkten verbessert.

Zur Freude des Verfassers hat sein Buch auch im Auslande Beifall gefunden, wie das Erscheinen einer englischen Übersetzung beweist. Eine Übersetzung ins Russische befindet sich unter der Presse.

Heidelberg, im Februar 1900.

Gattermann

Vorwort zur funften Auflage.

Das vorliegende Buch ist ein unveränderter Abdruck der vierten Auflage. Mögen die Freunde des Büchleins demselben auch im alten Gewande treu bleiben.

Freiburg, im Oktober 1901.

Gattermann

\title{
Vorwort zur sechsten Auflage.
}

Dem Charakter des Buches entsprechend wurden in die neue Auflage zwei Beispiele für die so fruchtbare Grignardsche Reaktion neu aufgenommen. An den alten Vorschriften etwas zu ändern, erwies sich als nicht notwendig.

Freiburg, im Oktober 1903.

Gattermann

\section{Vorwort zur siebenten Auflage.}

Die neue Auflage unterscheidet sich von den früheren dadurch, daB theoretische Erörterungen, die für das organischpräparative Arbeiten von allgemeiner Bedeutung sind (Theorie der Wasserdampfdestillation, Teilungssatz, Massenwirkungsgesetz, Löslichkeitsprodukt), nach Möglichkeit Berücksichtigung fanden. Ferner wurde die Tabelle für die Berechnung der Stickstoffbestimmungen auf Grund des von RAYLEIGH und RAMSAX ermittelten Stickstoffgewichtes neu berechnet, bei welchem Anlasse auch die Mantissen der dekadischen Logarithmen eingefügt wurden.

Freiburg, im April 1905.

Gattermann 


vir $\quad$ Vorwort

\section{Vorwort zur achten Auflage.}

Die achte Auflage ist im wesentlichen identisch mit der siebenten. Es wurden nur auf Grund praktischer Erfahrungen sowie neuerer wissenschaftlicher Publikationen im praktischen wie theoretischen Teile entsprechende Verbesserungen eingefügt. Von der Kritik war mir empfohlen, bei einer neuen Auflage auch die Dennstedtsche Methode der Elementaranalyse zu berücksichtigen. Ich erwidere darauf, da $B$ seit längerer Zeit in meinem Institute zwei DENNSTEDT-Öfen in Betrieb sind und daB damit vielfach gute Resultate erzielt wurden. In einer Reihe von Fällen versagte die Methode jedoch. Da auch von anderer Seite (B. 39, 1615) derartige Erfahrungen gemacht sind, so glaubte ich die Aufnahme des neuen Verfahrens in das vorliegende Buch so lange aufschieben zu sollen, bis die in Aussicht gestellte zweite Auflage (B. 39, 1623) der DenNSTEDTschen Anleitung erschienen ist. Freiburg, im November 1906.

Gattermann

\section{Vorwort zur neunten Auflage.}

$\mathrm{Zu}$ wesentlichen Veränderungen lag kein AnlaB vor. Verbessert wurden nur die Vorschriften für die Darstellung von Äthylen, Glykol und Kohlenoxyd.

Freiburg, im November 1908.

\section{Gattermann}

\section{Vorwort zur zehnten Auflage.}

Nachdem die Elementaranalyse nach DenNSTEDT sich in meinem Institute während eines Zeitraumes von fünf Jahren in den allermeisten Fällen glänzend bewährt und die Lrebiasche Methode fast ganz verdrä日gt hat, durfte ich nicht länger zögern, sie in dies Buch aufzunehmen. - Die übrigen Kapitel wurden nur einer gründlichen Durchsicht unterzogen.

Freiburg, im Oktober 1910.

Gattermann

Vorwort zur fünfzehnten Auflage.

Abgesehen von einigen Verbesserungen ist die vorliegende Auflage wie die vorhergehende ein unveränderter Abdruck der dreizehnten.

Freiburg, im Juni 1920.

Gattermann 